\title{
An evolutionary model for initiation, promotion, and progression in carcinogenesis
}

\author{
T.L. VINCENT ${ }^{1}$ and R.A. GATENBY ${ }^{2,3}$ \\ ${ }^{1}$ Aerospace and Mechanical Engineering, and Departments of ${ }^{2}$ Radiology and \\ ${ }^{3}$ Applied Mathematics, University of Arizona, Tucson, AZ 85721-0119, USA
}

Received November 9, 2007; Accepted January 7, 2008

\begin{abstract}
Human carcinogenesis is a multistep process in which epithelial cells progress through a series of premalignant phenotypes until an invasive cancer emerges. Extensive experimental observations in carcinogenesis have demonstrated this process can be divided into three general eras: initiation, promotion, and progression. However, this empirically derived, tissue-level explanation of carcinogenesis has not been reconciled with the step-wise genotypic and phenotypic changes encompassed in evolutionary paradigms such as the Feoron-Vogelstein diagram. Here, we analyze an evolutionary model of cellular dynamics that defines mutual interactions of cellular and subcellular events and tissue level changes in tumor growth and morphology. Results are expressed using an adaptive landscape that illustrates the evolutionary potential of cells that allow them to adapt to specific microenvironmental selection forces. It is shown that normal epithelial cells have a novel adaptive landscape that permits coexistence of normal cellular populations but also allows invasion by mutant phenotypes. Subsequent cancer evolution is possible due to a relaxation of tissue growth constraints (as mediated by cell-cell and cell-extracellular matrix interactions) and adaptations in response to perturbations in microenvironmental substrate concentrations (due to separation of evolving tumor cells from their blood supply by an intact basement membrane). Simulations, based on the dynamic model, produce three distinct stages of carcinogenesis that are consistent with the initiation, promotion, and progression stages observed experimentally. The simulations provide insight into the underlying cellular and microenvironmental dynamics that govern these empirical observations and suggest novel prevention strategies that may be tested experimentally.
\end{abstract}

Correspondence to: Dr Thomas L. Vincent, Aerospace and Mechanical Engineering, University of Arizona, Tucson, AZ 85721-0119, USA

E-mail: vincent@u.arizona.edu

Key words: somatic evolution, carcinogenesis, modeling cancer, evolutionary games, Darwinian dynamics

\section{Contents}

1. Introduction

2. Model of in vivo cellular dynamics

3. Adaptive landscape for normal cells

4. In vivo growth constraints and cellular adaptations

5. Numerical simulations

6. Carcinogenesis

7. Model summary

8. Discussion

\section{Introduction}

Empirical observations, primarily in experimental models of skin cancer, demonstrate carcinogenesis is divided into three distinct steps: initiation, promotion, and progression $(1,2)$. Initiation follows exposure to mutagens and results in little or no observable changes in the cellular or tissue morphology but does confer a permanent increase in susceptibility to cancer formation. Tumor promotion requires non-mutagenic tissue disruption by wounding or inflammation and results in formation of a non-malignant tumor which may regress without further stimulus. Subsequent tumor progression and malignant transformation requires some additional tissue disruption although much of cellular evolution in this phase seems to require no external stimulus. This conceptual model of carcinogenesis is now well documented experimentally and remains widely accepted. In 1986, Spandidos (3) first proposed a model linking these empirical observations with extant information regarding three classes of oncogenes. However, the underlying cellular and microenvironmental evolutionary dynamics that lead to these observable tissuelevel eras have not been clearly defined and reconciled with the multistep, genetically base models such as the FearonVogelstein diagram (4).

Here, we investigate a mathematical model for the microenvironment selection forces and cellular phenotypic adaptations during somatic evolution of epithelial cancers using methods from evolutionary game theory. Our goal is to understand the multi-scalar dynamics that transforms these molecular and cellular events into the macroscopic changes empirically observed in experimental models of carcinogenesis.

According to the model developed below, the earliest mutations during carcinogenesis affect tumor suppressor 
genes and produce minimal changes in the cellular phenotype with limited proliferation. However, these mutations alter the adaptive landscape, associated with the model, into one that permits subsequent evolution to an invasive cancer. This is identical to the changes observed following initiation in the classical experimental models of carcinogenesis (1) in which initiation only 'primes' the local tissue for subsequent development of cancer following additional stimuli such as wound or chronic inflammation. Furthermore, the model predicts that subsequent evolution of an invasive cancer entails two distinct components similar to the promotion and progression stages of observed in carcinogenesis experiments. The first is dominated by tissue-generated proliferation constraints and promotes adaptations that relax these proliferation barriers through mutations in oncogenes, tumor suppressor genes, and senescence pathways. The second phase is largely governed by microenvironmental factors such as blood flow and substrate diffusion that control local concentrations of oxygen, glucose, and extracellular $\mathrm{pH}$. In this component cellular proliferation is restricted by concentrations of oxygen, glucose, or $\mathrm{H}^{+}$. This promotes adaptations such as upregulation of glycolysis and resistance to acid-mediated toxicity that lead to final evolution of an invasive cancer. The cellular and genetic changes observed in the mathematical models of each era represent explicit predictions that can be tested experimentally.

\section{Model of in vivo cellular dynamics}

Under normal conditions, growth of epithelial cells is constrained by signals they receive from each other, the basement membrane and positive and negative growth factors secreted by the normal stromal (support) cells including blood vessels deep to the basement membrane. These constraints are represented by the $a$ and $K$ functions in the following tissue model. In addition, each cell requires a quantity of substrate $\mathrm{m}$ for maintenance of its basal functions so that cellular proliferation may occur only when substrate availability in excess of that quantity. The tissue model of Gatenby (5) was previously modified to put it in an evolutionary context (6). A new model is presented with some significant extensions. In particular, the new model: requires only a single adaptive parameter instead of two; explicitly includes tissue controls that incorporates heritable changes in tumor suppressor genes and oncogenes; has maximum substrate delivery rate adjusted according to demand rather than externally through manual changes; provides a mechanism by which mutant cells become acidic; accounts for the effect of an acidic environment on normal cells; provides a realistic relationship between the adaptive parameter and growth regulation through substrate uptake; does not allow the substrate uptake term to go negative.

Using the $G$-function method (7) for describing the fitness of any cell, the evolutionary cell model is given by:

$$
G(v, \mathbf{u}, \mathbf{x}, R)=B_{n}\left(1-\frac{\sum_{i=1}^{n_{s}} a_{j}(v, \mathbf{u}) x_{j}}{K(v)}\right) \operatorname{step}\left(\frac{E(v) R^{2}}{R_{0}^{2}+R^{2}}-m\right)
$$

were the fitness of any cell is obtained by replacing the virtual variable $v$ by the strategy that cell is using. The first term in parenthesis is the standard Lotka-Volterra competition equation. The second term in parenthesis regulates the intrinsic growth term, $B_{n}$ as a function of excessive substrate uptake where $m$ is basal demand. The step function does not allow this term to go negative by returning the value in parenthesis if it is positive and zero if the value in parenthesis is zero or negative.

It is assumed that there are $n_{s}$ different cell types (including both normal and mutant cells). The scalar strategies are adaptive parameters used by the different cell types and are denoted by the vector:

$$
\mathbf{u}=\left[\begin{array}{lll}
u_{1} & \cdots & u_{n_{s}}
\end{array}\right]
$$

with the density of the different cell types denoted by the vector:

$$
\mathbf{x}=\left[\begin{array}{lll}
x_{1} & \cdots & x_{n_{3}}
\end{array}\right]
$$

Here we assume the nutrient $R$ is glucose. Its uptake into the cell is primarily determined by the number and type of membrane glucose transporters (GLUT). Here we assume that GLUT expression as well as the level of anaerobic glucose metabolism is dependent on the intranuclear concentrations of hypoxia inducable factor (HIF). The intracellular concentrations of HIF in cells $x_{i}$ are assumed to be an adaptive parameter and are identified with the strategy variable $u_{i}$. The concentration of HIF is assumed to vary between zero and one and is thought of as a percentage of maximum available. The role of oxygen in proliferation is incorporated in the model through the carrying capacity (below). Oxygen enters the cell by simple diffusion so that the intracellular concentrations are influenced only by the concentration of oxygen in the environment. Substrate dynamics of glucose is presumed to follow Michaelis-Menten uptake:

$$
\dot{R}=r-\sum_{i=1}^{n_{s}} \frac{E(v) R^{2}}{R_{0}^{2}+R^{2}} x_{i}
$$

where $r$ is substrate delivery rate as given by:

$$
r=r_{e} m \sum_{i=1}^{n_{s}} x_{i}
$$

where $\mathrm{r}_{e}$ represents physiologic control of flow through vascular networks and must be $>1$ for cell proliferation (i.e. delivery must exceed basal demand). We assume maximum substrate delivery limited by local vascularity:

$$
\begin{aligned}
& r_{\max }=r_{m_{1}}+r_{m_{2}} \sum_{i=1}^{n_{s}} u_{i} x_{i} \\
& \text { if } r>r_{\max } \text { then } r=r_{\max }
\end{aligned}
$$

where $r_{m l}$ is the maximum flow rate in the absence of an external event (such as wounding) and $r_{m 2}$ is a constant that adjusts the increase in maximum flow level due to an external event. It is assumed that $r_{m l}$ is slightly larger than the normal substrate delivery rate, $r_{m l}>r_{e} m x^{*}{ }_{1}$, where $x^{*}{ }_{1}$ is the equilibrium population of normal cells. 


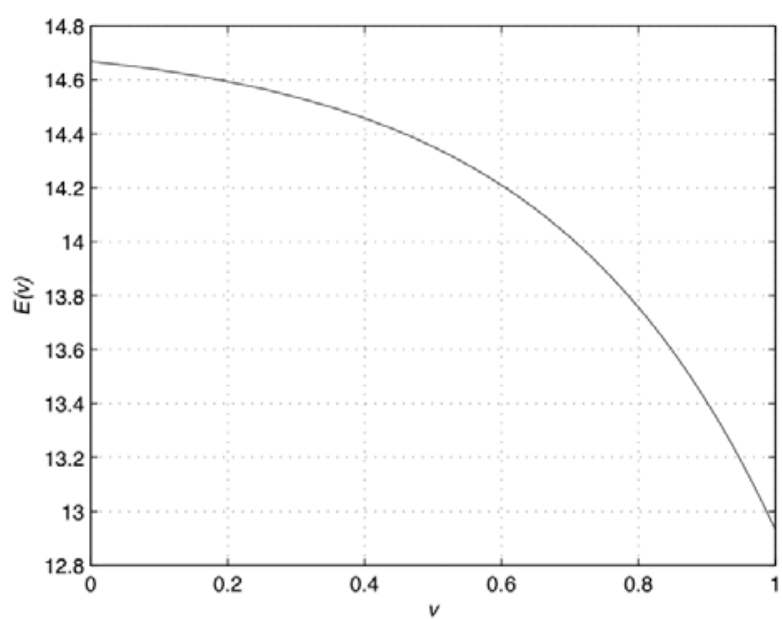

Figure 1. The substrate function $E(v)$ decreases as the percent of HIF goes to one.

In epithelial surfaces, blood flow carries substrate to the extracellular space adjacent to the basement membrane. From there glucose and oxygen must diffuse across the membrane to supply the epithelial cells. As the cells proliferate during the carcinogenesis process both the demand for substrate and the distance it must diffuse to reach the cells increases.

Both substrate delivery and the cellular interaction term are assumed to depend on $v$. Fig. 1 illustrates the function used to model the substrate delivery drop off with an increase in HIF concentration. The cellular interaction term is a function of the difference between the HIF concentration of a focal individual and any other mutant individuals in the population that are at a different HIF concentration as given by:

$$
a_{j}(v, \mathbf{u})=a_{\text {mean }_{j}} \exp \left(-\frac{\left(v-u_{j}\right)^{2}}{2 \sigma_{a}^{2}}\right)
$$

The term $a_{\text {meanj }}$ for normal cell depends on HIF concentrations in mutant cells as illustrated in Fig. 2. The mutant cells are able to adapt to the acid environment and it is assumed that $a_{\text {mean }}$ for these cells remains fixed at a constant value of $a_{\text {mean } 2}=0.9$.

The carrying capacity is influenced by a number of factors including cells size, local physical constraints, and substrate availability. Because oxygen concentrations decline more rapidly with distance than glucose [see discussion] it is viewed as the critical environmental substrate limiting intraductal tumor growth. This results in cellular necrosis in the ductal lumen frequently observed in pathological specimens.

Under physiologic conditions blood flow is regulated to maintain oxygen concentrations within a narrow range. Normal cells typically use aerobic metabolism of glucose which produces about 36 moles of ATP per mole of glucose. A switch to glycolysis (anaerobic metabolism that breaks down glucose to lactic acid rather than $\mathrm{H}_{2} \mathrm{O}$ and $\mathrm{CO}_{2}$ producing 2 moles of ATP per mole of glucose) can occur normally under conditions that produce low oxygen concentrations such as exercise, low blood pressure or in disease that interrupts

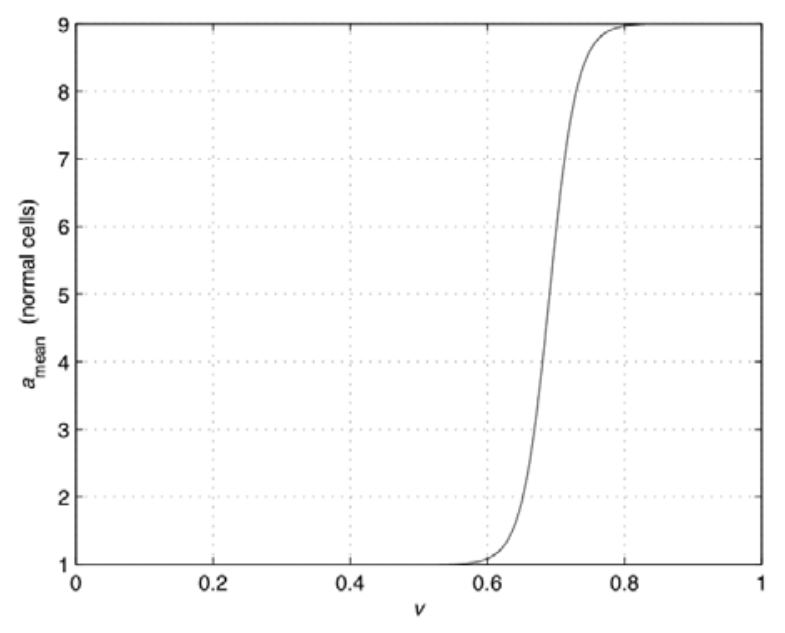

Figure 2. An acidic environment for normal cells greatly increases the mean value of $a$.

blood flow either acutely (e.g. stroke or myocardial infraction) or chronically (e.g. diabetic ulcers). Blood flow reduction to a tissue region results in hypoxia, cell death, and loss of tissue integrity.

In tumors, energy production from glycolysis is commonly observed. This is due in part to hypoxia caused by disordered vascular density and reduced flow. Tumor cells also characteristically demonstrate the Pasteur effect in which glycolytic pathways are utilized even in the presence of oxygen. The inefficiency of energy production by glycolysis is compensated by increased glucose uptake which is observed by FdG PET in the vast majority of human cancers $(9,10)$. However, tumor cells also characteristically exhibit the phenomenon of aerobic glycolysis. That is, they maintain inefficient anaerobic glucose metabolism even in the presence of oxygen. We include this effect in the model by assuming the carrying capacity (based on substrate metabolism) available to any single population of cells may be defined by the double humped function:

$K(v)=K_{\text {mean }_{1}} \exp \left(-\frac{\left(v-u_{K_{1}}\right)^{2}}{2 \sigma_{K}^{2}}\right)+K_{\text {mean }_{2}} \exp \left(-\frac{\left(v-u_{K_{2}}\right)^{2}}{2 \sigma_{K}^{2}}\right)$

The first hump at $v=u_{K I}$ corresponds to aerobic metabolism and the second hump at $v=u_{K 2}$ corresponds to anaerobic metabolism. In addition to inefficient metabolism, cells located at the right hump creates an acid environment. In general, cells using anaerobic metabolism excrete 5-fold more acid into the environment than those using aerobic metabolism (11). This results in a significant decrease in the local extracellular $\mathrm{pH}$. Normal cells exposed to an extracellular $\mathrm{pH}<7.1$ for more than a brief time will typically undergo necrosis or apoptosis. due to acid-induced increase caspase activity.

The way the model is set up, natural selection favors $v=1 / 3$ for the normal cells since this permits substantially greater ATP yield (i.e. 36 moles of ATP per mole of glucose vs. 2 for anaerobic metabolism) and does not produce an acid byproduct. When $v=1 / 3$, the cells are using an aerobic process for metabolism and when $v=2.5 / 3$ the cells are using an anaerobic process for metabolism. 


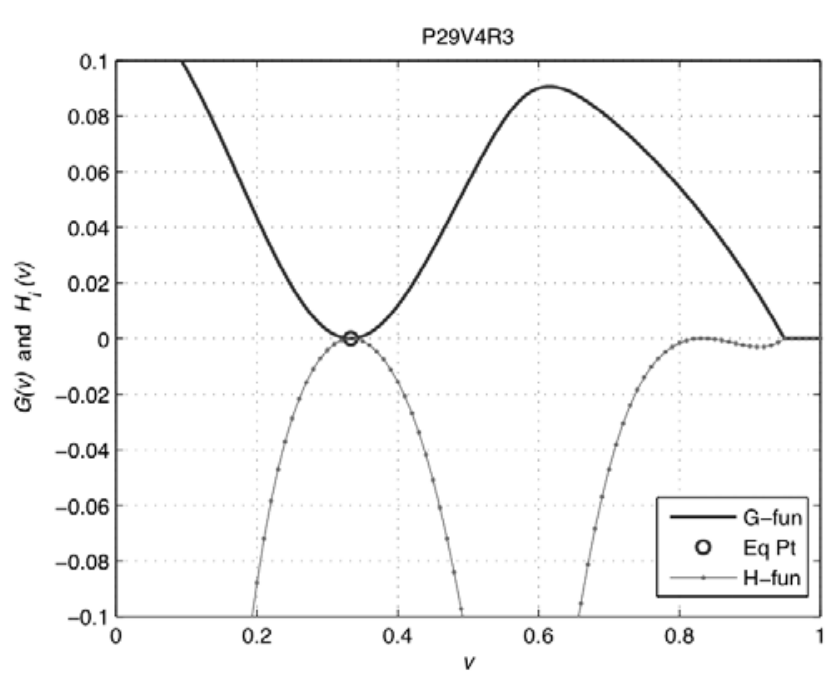

Figure 3. Under equilibrium conditions for $\mathbf{u}, \mathbf{x}$, and R, the normal cells lie at a minimum on the adaptive landscape but are at maximum fitness.

\section{Adaptive landscape for normal cells}

A plot of $G(v, \boldsymbol{u}, \boldsymbol{x}, R)$ vs. $v$ for fixed $\mathbf{u}, \mathbf{x}$, and $\mathrm{R}$ is called an adaptive landscape. Such a plot gives graphic information about the evolutionary stability of a particular strategy. Briefly, when $\mathbf{u}, \mathbf{x}$, and $\mathrm{R}$ are at equilibrium, an evolutionarily stable strategy (ESS) must correspond to a global maximum on the adaptive landscape (7). In a somewhat similar fashion, a plot $G\left(u_{i}, \boldsymbol{u}, \boldsymbol{x}, R\right)$ vs. $u_{i}$ for fixed $\mathbf{x}$ and $\mathrm{R}$ and with all strategies in $\mathbf{u}$ fixed except for $u_{i}$ graphically illustrates how the fitness of cells of type $i$ will change with strategy under the given conditions. We will plot both of these function together, designating the adaptive landscape plot by $G(v)$ and the fitness plot by $H_{i}(v)=\left.G\left(u_{i}, u, x, R\right)\right|_{u i=v}$ (replacing $u_{i}$ by $v$ in the $G$ provides a fitness function definition that can be plotted on the same graph with the $G$-function). In general, a plot of the adaptive landscape and a plot of a given fitness function do not coincide. For example, at equilibrium conditions, for normal cells, $v=1 / 3$ corresponds to a local minimum on the adaptive landscape but it is also a local maximum in fitness as illustrated in Fig. 3. This result is interpreted to mean that evolution maximizes cellular fitness within a stable tissue landscape. However, because the solution is not an ESS (i.e. not a global fitness maximum), other cell may coexist along side the normal cells. This feature allows for stable coexistence of multiple different cellular populations necessary for production of complex organs. However, while this configuration is necessary for organ formation it is also has the drawback that it allows for the invasion of an organ by mutant cell types. We will show that the development of cancer is a natural consequence of this particular landscape configuration. In other words, cancer is a 'price' that must be paid for multicellularity (6).

The reason the $G$-function plotted in Fig. 3 is at a local minimum is because, in the vicinity of this point, resources are not limiting and are available to mutant strategies that might be introduced at either side of the equilibrium solution.

The adaptive landscape illustrated in Fig. 3 was generated using the parameter values in Table I. This resulted in an equilibrium population of $x^{*}{ }_{1}=1000.9$ (not exactly 1000 due to the small effect of the second hump) with $r<\mathrm{r}_{\max }$ throughout the simulation run.

\section{In vivo growth constraints and cellular adaptations}

In equation (1), the in vivo cellular proliferation is constrained either by inhibitory interactions with other cells and the extracellular matrix or insufficient substrate availability. In normal tissue under physiologic conditions, the blood supply is assumed adequate to deliver substrate for proliferation. The proliferation of abnormal epithelial cells are physically

Table I. Nominal parameter values used in simulations.

\begin{tabular}{|l|l|l|}
\hline Parameter & Value & Units \\
\hline strategy at first hump in $K$ & $u_{K_{1}}=0.3333$ & dimensionless \\
\hline strategy at second hump in $K$ & $u_{K_{2}}=0.8333$ & dimensionless \\
\hline variance of humps & $\sigma_{K}^{2}=0.0178$ & dimensionless \\
\hline value of $K$ at first hump & $K_{\text {mean }_{1}}=1000$ & $10^{9}$ cells $/ \mathrm{cm}^{3}$ \\
\hline value of $K$ at second hump & $K_{\text {mean }}=1000$ & $10^{9}$ cells $/ \mathrm{cm}^{3}$ \\
\hline variance of interaction & $\sigma_{\alpha}^{2}=0.01$ & dimensionless \\
\hline flow rate control & $r_{e}=1.1$ & dimensionless \\
\hline maximum flow rate & $r_{m_{1}}=2820$ & micromoles glucose $/ 10^{6} \mathrm{cells} /$ day \\
\hline flow constant before external event & $r_{m_{2}}=0$ & micromoles glucose $/ 10^{6} \mathrm{cells} /$ day \\
\hline flow constant after external event & $r_{m_{2}}=1.2$ & micromoles glucose $/ 10^{6} \mathrm{cells} / \mathrm{day}$ \\
\hline basal demand & $m=2.544$ & micromoles glucose $/ 10^{6} \mathrm{cells} /$ day \\
\hline peak uptake & $E_{\text {max }}=14.668$ & micromoles glucose $/ 10^{6}$ cells $/$ day \\
\hline Michaelis-Menten factor & $R_{0}=3330$ & micromoles glucose $/ \mathrm{cm}^{3}$ \\
\hline intrinsic growth term & $B_{n}=0.5$ & cells $/$ micromole glucose \\
\hline initial substrate level & $R(0)=1702.9$ & micromoles glucose $/ \mathrm{cm}^{3}$ \\
\hline
\end{tabular}


constrained because they are not able to penetrate the basement membrane and must grow over each other into the potential space available within the lumen. When the cell numbers become sufficiently large, this can be observed as hyperplasia. The intact basement membrane serves to maintain a separation between the blood vessels and the hyperplastic intraductal tumor cells. As a result, oxygen, glucose, and $\mathrm{H}^{+}$must diffuse over increasingly longer distances as they pass from the blood vessels to the cells furthest from the basement membrane. It has been proposed that this will inevitably result in regions of hypoxia within the intraductal tumors (8). This produces a new era of carcinogenesis in which proliferation is constrained by local oxygen concentration. We will show below that if mutant cells initially lie to the right of the first hump in $K$ they will evolve toward the second hump. As they evolve closer to the second hump, they adapt to hypoxia by switching from aerobic to anaerobic metabolism. That is, in this environment, adoption to anaerobic metabolism represents a successful adaptation to anoxia. This factor will not have a large influence on the number of normal cells until the tumor cells have evolved to the vicinity of the second hump in $K$ resulting in increased glycolysis and local extracellular acidosis.

Thus, carcinogenesis requires adaptations to both components of in vivo proliferative constraints: tissue growth signals conveyed by interactions with other cells and the extracellular matrix and microenvironmental concentrations of oxygen, glucose, and $\mathrm{H}^{+}$that are dependent on blood flow and substrate diffusion.

\section{Numerical simulations}

The results of two simulation runs are presented. The first assumes that a random mutation event produces a cell that is less responsive to normal tissue constraints but otherwise possesses a normal phenotype including a normal mutation rate that prevents further evolution in the near term. Because the normal cells are at a minimum on the adaptive landscape, the mutant phenotype is able to coexist and does slowly increase in numbers. This rate of growth is so slow that a simulation run time maybe arbitrarily chosen resulting in little change in the mutant tissue density. In this case the simulation is allowed to run for 500 days. The ending values for the number of mutant cells and normal cells are then used as initial values for the second run. The second run starts when some environmental perturbation such as wounding or infection allows the mutant cells to evolve. The mechanism by which the tissue perturbation increases in evolutionary rate include an increase in the mutation rate due to the clastogenic effects of injury or inflammation-induced hypoxia and acidosis and/or acquisition of defects in the DNA or chromosomal repair pathways. In addition the creation of harsh environmental selection pressures increase the slope of the adaptive landscape. The environmental perturbation could occur any time after the introduction of the mutant cells even up to several years later. In this era, a cellular phenotype evolves strategies to evade normal tissue growth constraints through further loss of tumor suppressor genes, gain of function mutations in oncogenes, and loss of normal senescence pathways. However, even though the mutant cells are now allowed to evolve, growth of these cells is eventually limited by insufficient substrate. This self-limiting cellular proliferation produces a 'benign' tumor. Finally, a second environmental perturbation is assumed to occur on day 175 of the second run. The nature of this external stimulus must be similar to the one seen in the previous era - wounding, infection or inflammation. However, at this point in the carcinogenesis process, the primary effect is due to increased substrate delivery. That is, because of the hyperemia induced by tissue injury, there is an increase in maximum substrate delivery. This allows for further evolution and growth of the mutant cells. Day 175 was chosen to impose the second perturbation because from this day onward the number of mutant cells would not appreciably change in number otherwise. The second run lasts for a total of 500 days ending with emergence of an invasive cancer.

The simulation runs, based on the above model, are obtained by integrating the following set of differential equations:

$$
\begin{array}{r}
\dot{x}_{i}=\left.x_{i} G(v, \mathbf{u}, \mathbf{x}, R)\right|_{v=u_{i}} \\
\dot{R}=r-\sum_{i=1}^{n_{s}} \frac{E(v) R^{2}}{R_{0}^{2}+R^{2}} x_{i} \\
\dot{u}_{i}=\left.\sigma_{i}^{2} \frac{\left.\partial G(v, \mathbf{u}, \mathbf{x}, R)\right|_{v=u_{i}}}{\partial v}\right|_{v=u_{1}}
\end{array}
$$

with $G(v, \boldsymbol{u}, \boldsymbol{x}, R)$ given by (1). The first equation describes the population dynamics in terms of the $G$-function. The second equation is the same as (2) and the third equation describes

that strategy dynamics in terms of the $G$-function (7). The $\sigma_{i}^{2}$ term is related to the variance in the distribution of

strategies available to the cells during reproduction. This term is set equal to zero for normal cells. All other equations used to define the $G$-function are given above with nominal values for the parameters given in Table I.

\section{Carcinogenesis}

Simulations of somatic evolution of cancer based on our model produces three distinct eras very similar to initiation, promotion, and progression as illustrated in Fig. 4. The first part of the initiation era corresponding to the introduction of a mutant and its slow growth up to the first perturbation event and is not plotted. This period of time is indefinite with very limited mutant growth. Thus time $t=0$ in Fig. 4 corresponds to the time of the first perturbation event that allows evolution to take place during initiation. The top panel plots the density of both the normal and mutant cells. During this part of the initiation phase, the mutant cells proliferate very slowly and eventually reach maximum. This is accompanied with a very slight drop in the density of the normal cells. The main effect of this phase is a change in the shape of the adaptive landscape that permits evolution toward a more malignant population. However, this is initially prevented by the maximum substrate delivery rate. This simulation result is virtually identical to the initiation phase observed in experimental carcinogenesis models. Simulations demonstrate that further tumor development will occur only if environmental conditions change to allow further evolution. Our 

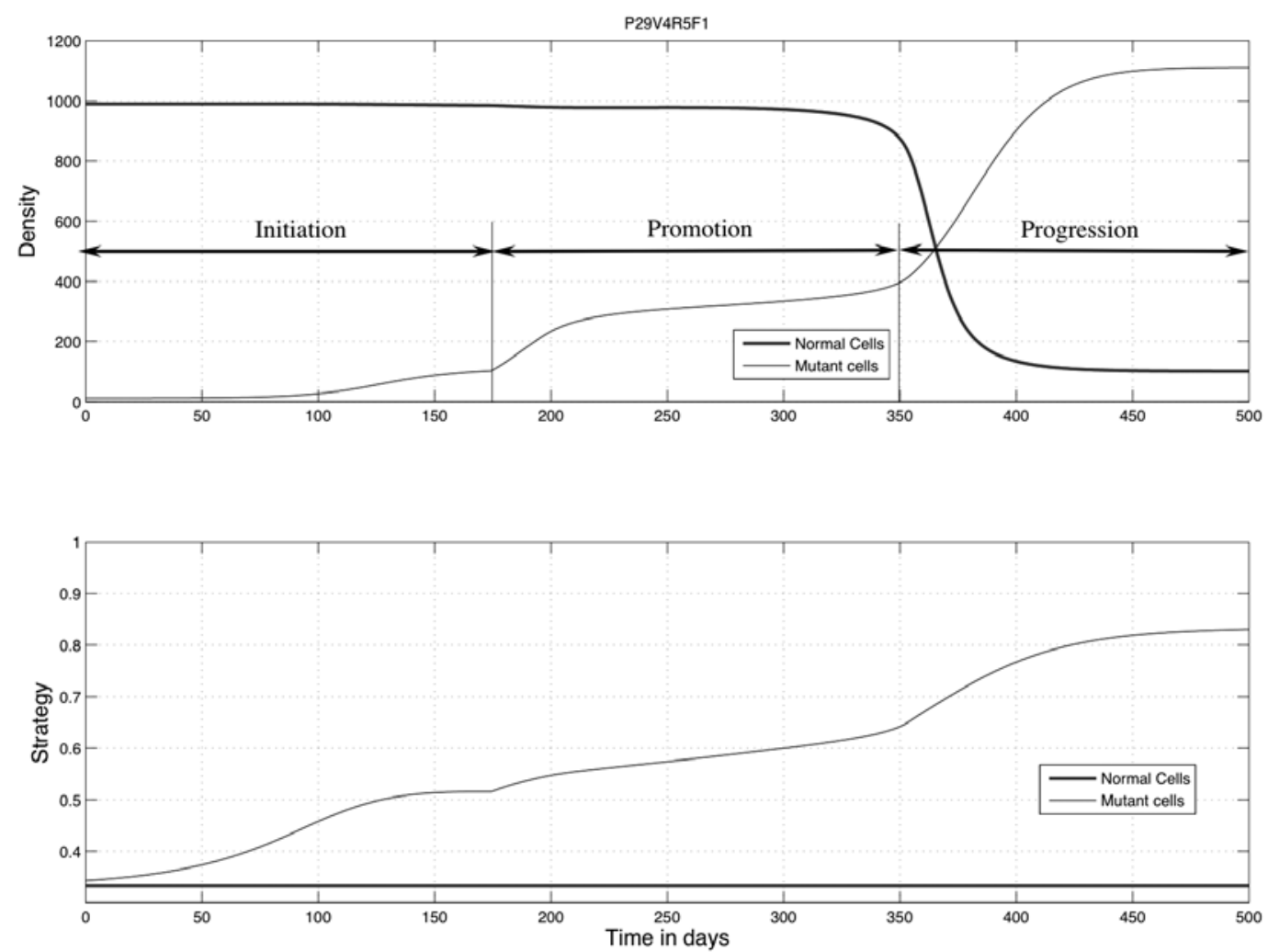

Figure 4. Simulation of the three eras of carcinogenesis.
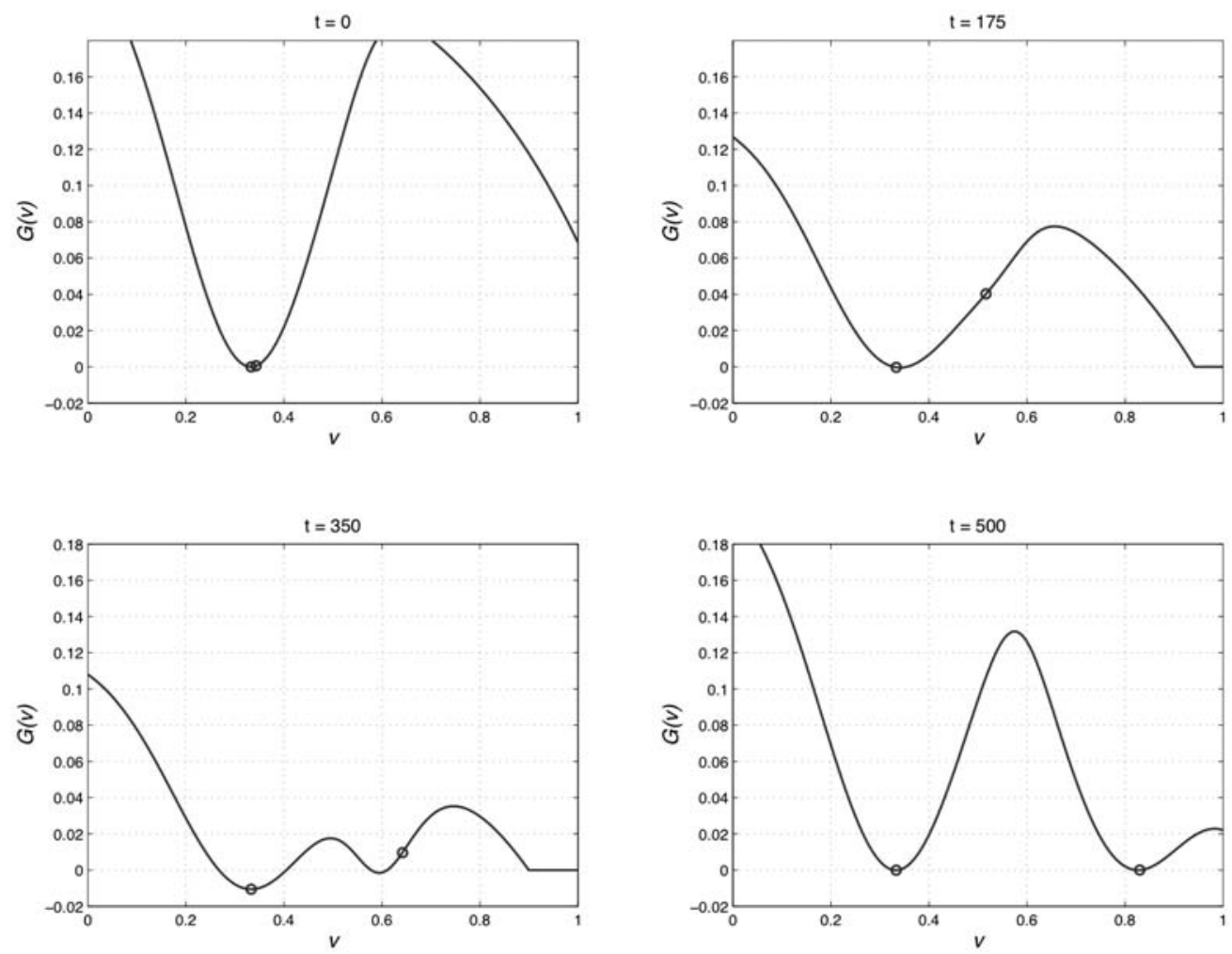

Figure 5. Changes in the adaptive landscape during the three eras of carcinogenesis.

results suggest that the primary effect of tumor promoters is an increase in the evolution rate (probably due to clastogentic effects of hypoxia and acidosis or an increase in selection pressures due to harsh environmental conditions) which permits further evolution to more malignant phenotypes. The simulations demonstrate that the phenotypic changes that 


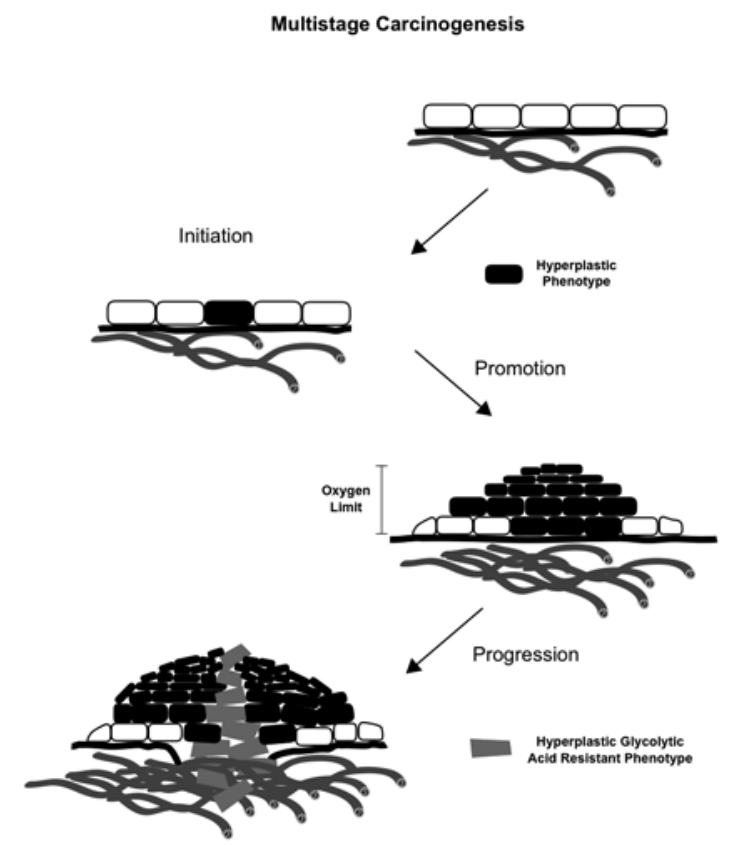

Figure 6. This figure illustrates multistage carcinogenesis based on empirical observations (primarily in skin cancer). The initiation step follows exposure to a mutagen such as X-rays and results in a heritable cellular change that does not significantly change the cellular or tissue morphology but does confer a long-term increase in risk of cancer development. The promotion stage requires a non-mutagenic stimulus such as chronic inflammation. This enhances cellular proliferation and, in our model, creates local increased vascular density and blood flow. The promotion step results in formation of a localized tumor that displays non-malignant, self-limited growth and may regress if the promoting stimulant is withdrawn. We propose that further growth in this lesion is limited by diffusion limit of oxygen as the tumor expansion carries the proliferating cells further from the blood vessels from which they remain separated by an intact basement membrane. The consequent regional hypoxia and acidosis produce a toxic environment that limits further proliferation. In the final progression step, the tumor transitions to limitless, invasive growth. Model simulations demonstrate that this is the result of adaptation to the hypoxic, acidic environment that limited growth after promotion. The consequent phenotype is both glycolytic and resistant to acid-mediated toxicity. The model simulations demonstrate this phenotype is invasive because it produces an acidic environment through upregulation of glycolysis that is toxic to other cellular populations but not itself. evolve during the promotion era are primarily adaptations to overcome tissue control factors and include additional mutations in tumor suppressors, oncogenes, and senescence pathways. During the promotion phase the mutant cells move to a plateau of higher density without a significant drop in the density of the normal cells. Thus, tumor growth in the promotion era remains self-limited and, therefore, nonmalignant. We find the reason for this limitation in tumor growth is emergence of growth limitation due to a decline in substrate concentrations as proliferation away from the basement membrane increases the distance of mutant cells from the underlying blood supply. The development of regions of hypoxia and acidosis promote cellular adaptations that lead to the progression phase in which the mutant cells move to another plateau of much higher density with a rapid drop in the density of the normal cells. Fig. 4 illustrated how the strategy of the mutant cells change during the three eras. The corresponding evolution of the mutant cells on the adaptive landscape is shown in Fig. 5 at four different time periods during the simulation run. They illustrate the configuration of the landscape at the beginning of initiation, at the beginning of promotion, at the beginning of progression, and at the end of progression. The mutant cells are always climbing a hill on the adaptive landscape, until the very end where a valley that has formed by panel 3 moves under the strategy in panel 4 .

Initiation. Initiation starts with a mutation that reduces the cell's sensitivity to tissue growth constraints. The starting point $(t=0)$ of the simulation results shown in Fig. 4 corresponds to the first environmental perturbation that allow the mutant cells to evolve. The initiated population, although creating no observable change in the appearance of the tissue, is nevertheless at an advantage over normal tissue because it sits on an adaptive landscape that permits evolution toward fitter and, therefore, more tumorigenic phenotypes. This is consistent with experimental observations that show tumor initiation is the result of a mutation event but this transformed population produces minimal or no observable effect on the

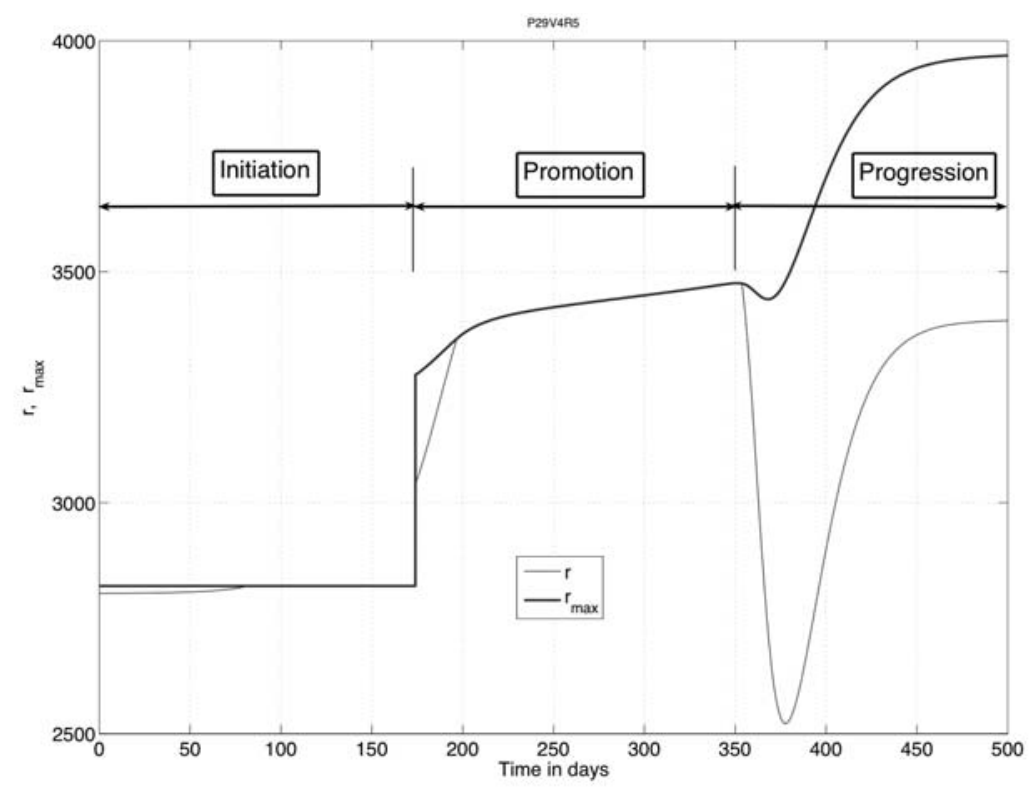

Figure 7. Mathematical modeling replicates the empirical observations of Fig. 6. 
morphology of the tissue and will not, without additional stimuli, form a tumor. Although this initial event is not tumorigenic, the fact that the mutant cells now lie on a slightly positive slope of the adaptive landscape (Fig. 5, panel 1) introduces the potential for natural selection to operate on the mutant cells to drive them further into the initiation phase. As illustrated in Fig. 4 the mutant cells slowly increase in number and then rise to a quasi-equilibrium that can last for an indefinite length of time. The mutant cells while still small in number are beginning to line the basement membrane as shown by the dark cell in Fig. 6 .

Promotion. Experimental evidence demonstrates that, after initiation, tumor formation is stimulated by an external event such as wounding or inflammation (12). Our results similarly demonstrate that the initiation event will not be tumorigenic without introduction of a second environmental perturbation that increases the maximum substrate delivery rate. It allows cellular evolution presumably through the clastogenic effects of hypoxia and acidosis that accompanies wounding and inflammation or by creating a harsh environment that increases selection pressures. This event is simulated by replacing $r_{d}=0$ with $r_{d}=1.2$ at $t=175$ making these cells well positioned for further evolution and growth (Fig. 5, panel 2). However, during promotion, nutrients available to the cells are limited by $r_{\max }$ as illustrated in Fig. 7 and saturation of the substrate delivery rate to the tumor cells greatly slows down further evolution of the tumor. As a result, tumor growth during promotion eventually plateaus due to insufficient substrate. Cellular evolution during this time primarily involves adaptations to tissue growth constraints presumable through accumulating mutations in oncogenes, tumor suppressor genes, and senescent pathways. As a result of these changes, the mutant cells become quite numerous and have grown far into the lumen of the duct. Cellular adaptations take place as cells begin to switch to anaerobic metabolism. This is depicted in Fig. 6 with the anaerobic cells shown dark.

Our model suggests the primary mechanisms for tumor promotion are an increase in the evolutionary rate followed by an increase in local blood flow that alters the adaptive landscape. The former allows the cells to adapt to tissue proliferation constraints and promotes phenotypes that are increasingly insensitive to these constraints due, for example, to heritable changes in oncogenes and tumor suppressor genes. The latter represents a novel finding and previously unrecognized potential mechanism for tumor promoters. Indeed, current conceptual models which propose tumor promoter act through increased cellular proliferation and the potential role of increased evolutionary rate and alterations in blood flow and substrate delivery have not been previously considered $(1,2)$.

Progression. Although tumor growth continues following initiation and promotion, the basement membrane remains intact (breeching the integrity of the basement membrane defines the transition between premalignant lesions such as DCIS and invasive cancer). Since blood vessels remain deep to the basement membrane, substrate must diffuse over increasingly long distances resulting in severe hypoxia in those cells $>100$ microns (about 5 cell layers) from the membrane $(13,14)$. As $r_{\max }$ continues to increase, the tumor cells are able to eventually evolve close to the peak of the right hand hump in $K$. This increases the fitness of the mutant cells as they adapt to their own acid-induced toxicity. In addition, the effect of this toxicity on the normal cells as determined from Fig. 2 results in a large die off of these cells (often visualize in pathologic specimens as intraductal necrosis). Once $r_{\max }$ is large enough for the tumor cells to evolve past the steep rise in the acid factor, evolution progresses very rapidly. The reason for this is due to the rapid die off of normal cells resulting in a larger substrate delivery rate to the tumor cells. The tumor has now achieved sufficient size to breech the basement membrane and form an invasive cancer as illustrated in Fig. 6.

We find that the progression phase of carcinogenesis is dominated by cellular adaptations to harsh microenvironmental conditions including hypoxia and acidosis. The phenotype that emerges from this era has a potent proliferative advantage because it creates an acidic environment (through upregulated glycolysis) that is toxic to its competitors but not itself. This results in a large die off of competing phenotypes that we propose is essential for evolution of an invasive cancer.

\section{Model summary}

The simulations demonstrate that the first steps in carcinogenesis must involve mutations in tumor suppressor genes and will produce no observable changes in the cellular phenotype and tissue architecture. This result reproduces the initiation phase of experimental carcinogenesis and is consistent with the Fearon-Vogelstein model in which a tumor suppressor mutations (APC mutation) is the first step in carcinogenesis. This era is static because the underlying cellular changes do not permit evolution. The second phase of carcinogenesis occurs only after a tissue perturbation that permits cellular evolution and will consist of changes in tumor suppressor genes and oncogenes which reduce the tumor cell response to normal tissue proliferation constraints. This results in self-limited tumor growth and is consistent with the promotion era of carcinogenesis as well as the mutations observed in the later stages of the Fearon-Vogelstein. An invasive cancer emerges only after a third era in carcinogenesis dominated by increased substrate delivery and evolution of the glycolytic and acid-resistant phenotypes. This era is similar to the progression phase observed in carcinogenesis but has no equivalent set of changes in the Fearon-Vogelstein model. Here the model explicitly predicts, for example, that the glycolytic phenotype (with corresponding increase in glucose metabolism) should be absent during the promotion stage but emerge during the progression era.

\section{Discussion}

We model the multistep process of carcinogenesis using evolutionary game theory. Our model incorporates microenvironmental selection forces and cellular adaptations within the geometrical constraints imposed by the anatomy of epithelial surfaces on which premalignant tumors evolve. The results provide a theoretical framework for the cellular and extracellular dynamics that govern somatic evolution 
during the initiation, promotion, and progression eras of carcinogenesis. While there is abundant literature on the molecular and cellular events during carcinogenesis, up to now there has been no consensus mutiscalar theoretical model that integrates the somatic evolution concept of carcinogenesis with the experimentally observed, tissue-level changes of initiation, promotion and progression.

The main findings and predictions of the mathematical model are: i) Functioning healthy tissue possesses an adaptive landscape that allows coexistence of non-evolving normal cell populations - a condition necessary for multicellular organisms. However, it also permit invasion by fitter phenotypes and, therefore, tumor development. In other words, the potential for carcinogenesis is a 'penalty' incurred as the consequence of a tissue landscape that permits multicellular function. ii) Tumor initiation results from mutations that weaken local tissue growth constraints. The effect of this mutation is not due to population proliferation but rather a change in the configuration of the normal tissue adaptive landscape such that the mutant population exists near a fitness minimum. This results in cellular growth given a subsequent perturbations that allows for evolution. iii) Tumor promoters such as wounding or inflammation, not ordinarily tumorigenic, can promote tumor growth by increasing local blood flow. This differs from the conventional assumption that promotion acts by increasing cellular proliferation. An explicit prediction is that antiangiogenesis therapy will significantly inhibit tumor promotion. This suggests a previously unrecognized potential role for angiogenesis inhibition as a tumor prevention strategy. iv) Following the promotion step, tumor growth is eventually limited by substrate limitation. This is caused by the anatomic constraints imposed by intraductal growth and leads to a sequence of events including regional hypoxia, upregulations of glycolysis, microenvironmental acidosis and cellular adaptation to acid-induced toxicity. v) This sequence of development of substrate limited environment, cellular adaptation to hypoxia and then acidosis is critical for evolution of the malignant phenotype since the adaptive advantage confers the ability to destroy other populations. We propose this is a necessary final step in carcinogenesis and is observed in experimental systems as the progression era.

These results suggest possible new prevention strategies that can be tested experimentally. These include: i) Modifying environmental factors in the adaptive landscape could possibly stop tumor growth. For example, if the landscape located at the tumor strategy value could be changed from a positive to a negative slope, evolution would tend to move the tumor cell strategy back toward aerobic metabolism. This might be accomplished by modifying the microenvironment of the tumor. For example, altering the $\mathrm{pH}$ content of the lumen of the colon could alter the selection pressures in the adaptive landscape. Similarly, induction of brief systemic acidosis (by vigorous exercise, for example) might sufficiently alter the environment in premalignant lesions to slow or even reverse the evolutionary process. ii) Pharmacological alterations in cellular adaptations to the predicted sequence of environmental changes may block evolution of the malignant phenotype. For example, drugs that block the $\mathrm{Na}^{+} / \mathrm{H}^{+}$antiport may reduce the ability of cells to adapt to acidic environments and halt the evolutionary process.

\section{References}

1. Rubin H: Microenvironmental regulation of the initiated cell. Adv Cancer Res 90: 1-62, 2003.

2. Hennings H, Glick AB, Greenhaigh DA, Morgan DL, Strickland JE, Tennenbaum T and Yuspa SH: Critical aspects of initiation, promotion, and progression in multistage epidermal carcinogenesis. Proc Soc Exp Biol Med 1: 1-8, 1993.

3. Spandidos DA: A unified theory for the development of cancer. Biosci Rep 6: 692-708, 1986.

4. Fearon ER and Vogelstein B: A genetic model for colorectal tumorigenesis. Cell 61: 759-767, 1990.

5. Gatenby RA: Population ecology issues in tumor growth. Cancer Res 51: 2542-2547, 1991.

6. Gatenby RA and Vincent TL: An evolutionary model of carcinogenesis. Cancer Res 63: 6212-6220, 2003.

7. Vincent TL and Brown JS: Evolutionary Game Theory, Natural Selection, and Darwinian Dynamics. Cambridge University Press, Cambridge, 2005.

8. Gatenby RA and Gilles RJ: Why do cancers have high aerobic glycolysis? Nat Rev Cancer 4: 891-899, 2004.

9. Czernin J and Phelps ME: Positron emission tomography scanning: current and future applications. Annu Rev Med 53: 89-112, 2002.

10. Gambhir SS: Molecular imaging of cancer with positron emission tomography. Nat Rev Cancer 2: 683-693, 2002.

11. Schornack PA and Gillies RJ: Contributions of cell metabolism and $\mathrm{H}^{+}$diffusion to the acidic $\mathrm{pH}$ of tumors. Neoplasia 5: 135-145, 2003.

12. Balkwill F, Charles KA and Mantovani A: Smoldering and polarized ináammation in the initiation and promotion of malignant disease. Cancer Cell 7: 211-217, 2005.

13. Thomlinson RH and Gray LH: The histological structure of some human lung cancers and the possible implications for radiotherapy. Br J Cancer 9: 539-549, 1955.

14. Helmlinger G, Yuan F, Dellan $M$ and Jain RK: Interstitial pH and $\mathrm{pO} 2$ gradients in solid tumors in vivo: high-resolution measurements reveal lack of correlation. Nat Med 3: 177-182, 1997. 